\title{
FACTORES ASOCIADOS AL USO DE SERVICIOS DE SALUD ORAL EN ADULTOS MAYORES PERUANOS: ANÁLISIS SECUNDARIO DE ENCUESTA POBLACIONAL, 2018
}

\author{
Diego Azañedo ${ }^{1, a}$, Rodrigo Vargas-Fernández²,b , Carlos Rojas-Roque ${ }^{3, c}$
}

\begin{abstract}
RESUMEN
Objetivos. Identificar la prevalencia y factores asociados al uso de servicios de salud oral en adultos mayores (AM) peruanos durante el 2018. Materiales y métodos. Análisis secundario de los datos de 4874 AM peruanos de la Encuesta Demográfica y de Salud Familiar (ENDES) 2018. Se consideró el uso de servicio de salud oral (sí/no) en razón de los seis meses previos a la aplicación de la encuesta como variable dependiente, las variables independientes fueron: sexo, edad, área de residencia, nivel educativo, estado civil, dominio geográfico, limitación física, afiliación a un seguro de salud, lengua hablada y quintil de bienestar. Se realizó un análisis descriptivo usando frecuencias absolutas y proporciones ponderadas, y un análisis multivariado empleando modelos lineales generalizados (familia Poisson). Resultados. Del total de AM, 52,6\% fueron mujeres, 52,9\% pertenecían al grupo de 60 a 69 años de edad, $77 \%$ pertenecían al área urbana y $81,1 \%$ estuvieron afiliados a un seguro de salud. La prevalencia del uso de servicios odontológicos durante los últimos seis meses fue $24,9 \%$. El análisis multivariado encontró asociación con el área de residencia $(p<0,001)$, el nivel educativo superior $(p=0,001)$, la afiliación a un seguro de salud $(p<0,001)$, el dominio geográfico $(p=0,019)$ y todos los quintiles de bienestar $(p<0,001)$. Conclusiones. La prevalencia de uso de servicios de salud oral en AM fue baja, y sus factores asociados fueron el área de residencia, el nivel educativo, la afiliación a un seguro de salud, el dominio geográfico y los quintiles de bienestar.
\end{abstract}

Palabras clave: Salud Bucal; Anciano; Accesibilidad a los Servicios de Salud; Perú (fuente: DeCS BIREME).

\section{FACTORS ASSOCIATED WITH THE USE OF ORAL HEALTH SERVICES IN OLDER PERUVIAN ADULTS: SECONDARY ANALYSIS OF POPULATION SURVEY, 2018}

\begin{abstract}
Objectives. To identify the prevalence and factors associated with the use of oral health services in Peruvian older adults (OA) during 2018. Materials and Methods. Secondary analysis of data on 4,874 Peruvian OAs from the Demographic and Family Health Survey (ENDES) carried out in 2018. The use of oral health services was considered (yes/no) based on the six months prior to the application of the survey as a dependent variable. The independent variables were sex, age, area of residence, educational level, marital status, geographical domain, physical limitation, health insurance, spoken language, and welfare index quintile. A descriptive analysis was performed using absolute frequencies and weighted proportions, and a multivariate analysis using generalized linear models (Poisson regression). Results. From the total of OAs, $52.6 \%$ were women, $52.9 \%$ belonged to the $60-69$ age group, $77 \%$ belonged to the urban area, and $81.1 \%$ had a health insurance. The prevalence of the use of dental care services in the last six months was $24.9 \%$. Multivariate analysis found association with area of residence $(p<0.001)$, higher educational level $(p=0.001)$, health insurance affiliation $(p<0.001)$, geographic domain $(p=0.019)$, and all welfare index quintiles $(p<0.001)$. Conclusions. The prevalence of the use of dental care services in OAs was low, and its associated factors were the area of residence, educational level, health insurance, geographic domain, and welfare index quintiles.
\end{abstract}

Keywords: Oral health; Aged; Health services accessibility; Peru (source: MeSH NLM).

\footnotetext{
Universidad Católica Los Ángeles de Chimbote, Instituto de Investigación. Chimbote, Perú.

Universidad Científica del Sur. Lima, Perú.

CIDS, Centro de Investigación en Demografía y Salud. León, Nicaragua.

Cirujano dentista; ${ }^{\mathrm{b}}$ estudiante de Medicina; ${ }^{\mathrm{c}}$ licenciado en Economía

Recibido: 06/08/2019 Aprobado: 30/10/2019 En línea: 03/12/2019
}

Citar como: Azañedo D, Vargas-Fernández R, Rojas-Roque C. Factores asociados al uso de servicios de salud oral en adultos mayores peruanos: análisis secundario de encuesta poblacional, 2018. Rev Peru Med Exp Salud Publica. 2019;36(4):553-61. doi: 10.17843/rpmesp.2019.364.4724. 


\section{INTRODUCCIÓN}

El Perú atraviesa un proceso de envejecimiento poblacional y se estima que para el 2050 la proporción de adultos mayores (AM) alcanzaría el $22 \%$ del total de sus habitantes ${ }^{(1)}$. Esta etapa de la vida se caracteriza por presentar con mayor frecuencia problemas de salud oral, como caries dental, enfermedad periodontal y edentulismo parcial o total, afectando la calidad de vida de la persona, por lo que es necesario que reciban atención integral y oportuna por parte de los servicios de salud ${ }^{(2-4)}$. Además, estas condiciones orales no tratadas pueden contribuir a la generación de desigualdades a nivel social, económicas y del sistema de salud ${ }^{(5,6)}$. Para reducir estas desigualdades, el acceso a los servicios de salud oral de la población es de vital importancia, no sólo en términos de cobertura, sino también de acceso efectivo ${ }^{(7)}$.

Con la implementación de la ley delAseguramiento Universal de Salud (AUS), y el Plan Esencial de Aseguramiento en Salud (PEAS) ${ }^{(8)}$, se reconoce dentro de las prestaciones mínimas financiadas a tratamientos preventivos primarios (tales como profilaxis, colocación de sellantes y aplicación de flúor) y preventivos secundarios (como restauraciones, endodoncias y exodoncias). Sin embargo, no se ofrecen tratamientos rehabilitadores, a pesar de que el edentulismo es uno de los principales problemas de salud oral y está directamente asociado con la edad, más aún en aquellos con condición económica baja ${ }^{(9,10)}$. En ese sentido, la única opción de acceder a tratamientos de rehabilitación oral para personas adultas mayores en situación de pobreza y pobreza extrema, era el plan de intervención «Vuelve a Sonreír», que funcionó por el periodo 2012-2016 ${ }^{(11)}$, reactivándose a mediados del $2019{ }^{(12)}$. Dicho periodo de inactividad, pudo disminuir las posibilidades de acceso a estos tratamientos en AM.

Existen factores relacionados al uso de los servicios de salud más allá de la afiliación de la persona a un seguro de salud. A nivel mundial, estudios en AM, reportan que el ser mujer, tener mejor posición económica, mayor nivel educativo y estar afiliado a un seguro dental privado son factores que incrementan las probabilidades de acceder a los servicios de salud dental (13-15). En el Perú, en el 2014, un estudio en menores de 12 años identificó al estado socioeconómico, la región de residencia, el área de residencia, el grupo etario, el nivel educativo de los cuidadores y el tipo de servicio de salud como factores asociados al uso de servicios de salud oral ${ }^{(16)}$. Por otro lado, ese mismo año, el uso de servicios de salud oral en los últimos seis meses en AM peruanos alcanzó sólo $25 \%$ y la mayor parte de los atendidos lo hizo en servicios odontológicos privados de la costa y zonas urbanas ${ }^{(17)}$. La multidiversidad social, económica y cultural de la población peruana podría incidir sobre el uso a los servicios de salud oral, siendo importante su estudio para orientar la toma de decisiones destinadas a reducir las brechas de uso real en los grupos de población desprotegidos.

\section{MENSAJES CLAVE}

Motivación para realizar el estudio. En Perú no se dispone de información representativa y actualizada que evalúe los factores asociados al uso de los servicios de salud oral en adultos mayores (AM).

Principales hallazgos. Aquellos AM que vivían en la zona urbana, y en la selva, tuvieron menos probabilidad de utilizar los servicios odontológicos seis meses previos a la encuesta, además, los AM con nivel de educación superior, afiliados a un seguro de salud y con mayor quintil de bienestar tuvieron mayores probabilidades de uso.

Implicancias. Existen factores asociados al uso de servicios odontológicos en $\mathrm{AM}$ que deben ser considerados para la reducción de desigualdades en salud oral.

La Encuesta Demográfica y de Salud Familiar de Perú (ENDES), proporciona información descriptiva acerca de la prevalencia de uso de servicios odontológicos en AM según variables sociodemográficas; sin embargo, no se dispone de información representativa y actualizada que evalúe los factores asociados al uso de los servicios de salud oral en AM. Por ello, el objetivo del presente estudio fue identificar la prevalencia y factores asociados al uso de servicios de salud oral en AM peruanos durante el 2018.

\section{MATERIALES Y MÉTODOS}

\section{DISEÑO Y POBLACIÓN DE ESTUDIO}

Se realizó un análisis secundario de la información contenida en la ENDES 2018 realizada por el Instituto Nacional de Estadística e Informática (INEI) (http://iinei.inei. gob.pe/microdatos/). La ENDES recopila información de salud de los habitantes peruanos sobre hábitos de vida, salud materna e infantil, utilización de servicios de salud, enfermedades transmisibles y crónicas, entre otros. El tipo de muestra de la encuesta es bietápica, probabilística de tipo equilibrado, estratificada e independiente a nivel departamental y por área urbano rural. La unidad de muestreo y análisis son los hogares y las personas que residen en el hogar respectivamente. Se encuentran más detalles sobre el diseño de la muestra, la recopilación de datos y la calidad de los datos en el informe ENDES (http:// bit.ly/2wNiHOy). Para el presente estudio se emplearon las bases de datos de los cuestionarios de salud y hogar de la ENDES 2018.

Un total de 33794 personas mayores de 15 años con datos completos se incluyeron en el cuestionario de salud de la ENDES 2018. La muestra seleccionada para este estudio estuvo conformada por adultos de 60 años o más, definidos como AM según la legislación peruana ${ }^{(18)}$. Quedaron fuera del análisis 28920 personas por ser menores de 60 años. Los 4874 AM incluidos disponen de información completa de todas las variables consideradas en el estudio (Figura 1). 


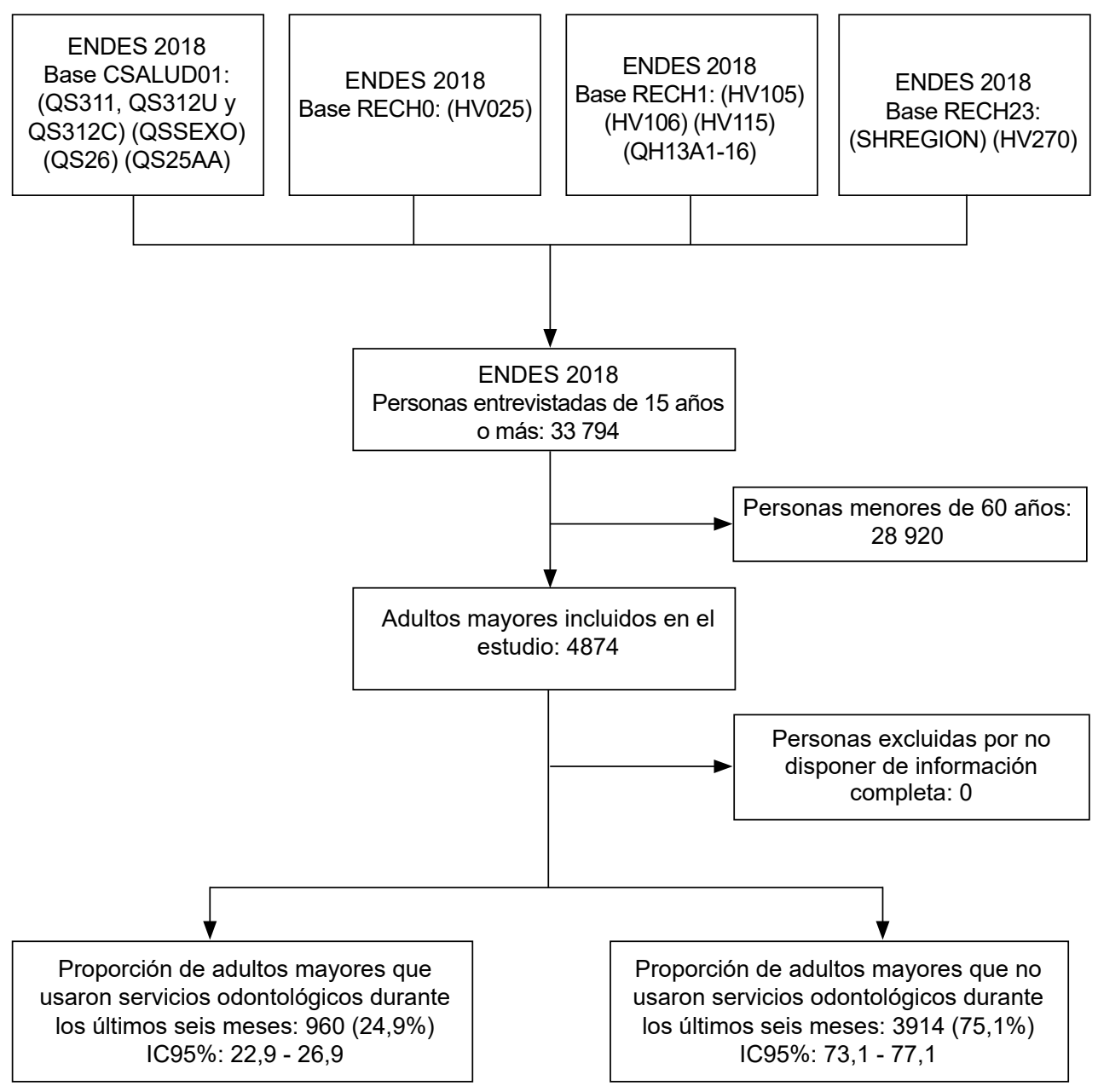

Figura 1. Flujograma de la selección de adultos mayores incluidos en el análisis, Encuesta Demográfica y de Salud Familiar (ENDES) 2018

\section{VARIABLES Y MEDICIONES}

Se consideró como variable principal de estudio el uso de los servicios de salud oral durante los seis meses previos a la encuesta (sí/no), recopilada mediante autorreporte y obtenida de las variables codificadas como QS311, QS312U y QS312C en el cuestionario de la ENDES 2018. Las variables independientes consideradas en el análisis, junto con sus códigos que aparecen en el cuestionario son las siguientes: sexo (hombre/ mujer) QSSEXO, edad (60-69/70-79/80-89/90 o más años), agrupada de la variable HV105, área de residencia (urbano/rural) HV025, nivel educativo (sin educación o educación inicial/primaria/secundaria/superior) HV106, estado civil (casado(a) o conviviente/soltero/viudo(a) o separado(a)) recodificada de la HV115, dominio geográfico (Lima Metropolitana/resto de Costa/Sierra/ Selva) SHREGION, limitación física (sí/no) construida a partir de preguntas sobre limitaciones para moverse, ver, oír, hablar, entender o relacionarse (QH13A1-A6), afiliación a un seguro de salud (sí/no) QS26, lengua hablada (quechua y lenguas aborígenes/ otra) recodificada de la variable QS25AA y quintil de bienestar (quintil inferior, segundo quintil, quintil intermedio, cuarto quintil, quintil superior) HV270. Las variables empleadas y sus códigos pueden ser identificados en el Sistema de Documentación Virtual de Investigaciones Estadísticas del INEI (https://bit.ly/2pj935U).

\section{ANÁLISIS ESTADÍSTICO}

Para el análisis se utilizó el software estadístico Stata $₫$ v14.2 (Stata Corporation, College Station, Texas, USA). Se especificaron las características del muestreo de la encuesta que incluyen las ponderaciones según estratos y diseño, mediante el comando $s v y$, las variables empleadas fueron HV001 (conglomerado), peso15_amas (factor de ponderación), y HV022 (estrato), usando el mismo comando se empleó la opción subpop () para realizar estimaciones de la subpoblación (personas adultas mayores de 60 
años). Las variables categóricas y la prevalencia del uso de servicios odontológicos fueron descritas mediante frecuencias absolutas y se estimaron las proporciones ponderadas con intervalos de confianza al 95\%. Para el análisis bivariado se empleó la prueba estadística de Chi cuadrado, fijando un valor de $\mathrm{p}$ de 0,05 .

Se usaron modelos lineales generalizados (GLM) de familia Poisson y función de enlace log tomando como variable dependiente el uso de los servicios de salud oral durante los últimos seis meses anteriores a la encuesta ${ }^{(19)}$. Para el modelo ajustado, se incluyeron aquellas variables que en el modelo crudo presentaron valor de $p<0,2{ }^{(20)}$. Se reportaron razones de prevalencia crudas y ajustadas (RP) con intervalos de confianza al $95 \%$, considerándose un valor de $p<0,05$ como estadísticamente significativo. Se evaluó la multicolinealidad entre las variables independientes medianteel comando collin deStata conel objetivo deobtener parámetros precisos en las estimaciones. En todos los casos el factor de inflación de la varianza fue menor a 10 por lo que se consideró una baja correlación entre las variables.

\section{CONSIDERACIONES ÉTICAS}

La realización del estudio no requirió la aprobación de un comité de ética por tratarse de un análisis de datos secundarios que son de dominio público y que no permite identificar a los participantes evaluados. Las bases de datos utilizadas en el presente estudio pueden obtenerse en la página web del INEI (http://iinei.inei.gob.pe/microdatos/).

\section{RESULTADOS}

La mayoría de los AM incluidos en el análisis fueron del sexo femenino $(52,6 \%$ ) y del grupo etario de 60 a 69 años de edad $(52,9 \%)$, media de 70,1 años (DE 7,81), tres de cada cuatro pertenecieron al área urbana y la mayoría de AM $(29,1 \%)$ alcanzó el nivel educativo primario. Más de la mitad de los AM estuvieron casados y ocho de cada diez estuvieron afiliados a un seguro de salud (Tabla 1).

La prevalencia del uso de servicios odontológicos durante los últimos seis meses fue de 24,9\%; IC95\%: 22,9-26,9. En AM residentes de áreas urbanas fue de 28,1\%; IC95\%: 25,730,7 , en AM con mayor nivel de educación fue de $45,8 \%$; IC95\%: $39,9-51,8 \%$, en afiliados a un seguro de salud fue de $26,8 \%$; IC95\%: 24,6-29,2, en aquellos que vivían en Lima Metropolitana fue de 34,5\%; IC95\%: 30,2-39,1, en aquellos con mayor nivel de bienestar fue de $42,1 \%$; IC95\%: $36,9-47,5 \%$, estos sub-grupos presentaron un mayor uso de servicios odontológicos que las demás categorías de las variables evaluadas $(p<0,001)$, asimismo, los AM que hablaban castellano, portugués $u$ otras lenguas extranjeras reportaron mayor porcentaje de uso a servicios odontológicos (26,6\%; IC95\%: 24,2-29,2) que aquellos que hablaban quechua y lenguas aborígenes (20,4\%; IC95\%: 17,3-23,9) ( $\mathrm{p}=0,005)$ (Tabla 1).

El modelo crudo reportó asociación entre el uso de los servicios odontológicos y todas las variables excepto el sexo, el grupo etario, la limitación física y estar soltero en referencia a ser casado/conviviente (Tabla 2). Sin embargo, en el modelo ajustado se encontró asociación con el área de residencia $(p<0,001)$, el nivel educativo superior en referencia a sin educación o inicial $(p=0,001)$, la afiliación a un seguro de salud ( $p<0,001)$, el dominio geográfico de la selva con respecto a Lima metropolitana $(p=0,019)$ y todos los quintiles de bienestar $(p<0,001)$ (Tabla 2).

El grupo área de residencia urbana tuvo menos probabilidades (RPa 0,62; IC95\%: 0,48-0,80) de uso de los servicios odontológicos que el grupo de área de residencia rural, y los AM que viven en la selva tuvieron menores probabilidades (RPa 0,73; IC95\%: 0,56-0,95) de uso que los que viven en Lima metropolitana. Los AM con nivel de educación superior (RPa 1,94; IC95\%: 1,33-2,83), con afiliación a algún seguro de salud (RPa 1,58; IC95\%: $1,23-2,03)$, y que están en un quintil de bienestar superior (quintil superior: RPa 3,84; IC95\%: 2,68-5,51), tienen más probabilidades de uso de servicios odontológicos en comparación a quienes tienen menor nivel de educación, no se encuentran afiliados a algún seguro o están en un quintil de bienestar inferior respectivamente (Tabla 2).

\section{DISCUSIÓN}

Nuestro estudio reportó que solo un $24,9 \%$ de los AM peruanos utilizaron los servicios de salud dental durante los seis meses previos a su participación en la ENDES 2018, observándose una reducción de 0,1\% referente al $25 \%$ reportado en el 2014 (17), lo que sugiere que los actuales programas y estrategias de salud oral en nuestro país no estarían mejorando las oportunidades de uso de los servicios de salud oral en esta población. Asimismo, se reportó que los AM con nivel de educación, los afiliados a algún seguro de salud y los de mayor quintil de bienestar tuvieron mayores probabilidades de uso de los servicios odontológicos que sus categorías de referencia; mientras que aquellos residentes del área urbana, y aquellos que viven en la selva, tuvieron menores probabilidades de uso de estos servicios. Así, las medidas de salud pública odontológica implementadas en el territorio nacional deben considerar las características asociadas con el uso de los servicios odontológicos con el objetivo de redireccionar recursos humanos y económicos a la población con mayor desventaja social y contribuir a la reducción de las desigualdades en el uso de estos servicios.

Se evidenció menores probabilidades de uso de servicios odontológicos en AM que residen en la zona urbana en comparación a quienes viven en la zona rural. Esta última concentra las regiones pobres de la sierra o selva 
Tabla 1. Características de los adultos mayores peruanos que usaron servicios odontológicos, Encuesta Demográfica y de Salud Familiar (ENDES) 2018

\begin{tabular}{|c|c|c|c|c|c|c|}
\hline \multirow{2}{*}{ Características } & \multicolumn{2}{|c|}{$\begin{array}{l}\text { Adultos mayores } \\
\text { encuestados }\end{array}$} & \multicolumn{3}{|c|}{$\begin{array}{l}\text { Uso de servicios odontológicos en } \\
\text { los últimos seis meses }\end{array}$} & \multirow[b]{2}{*}{ Valor de $p$} \\
\hline & $\mathrm{n}$ & $\begin{array}{l}\text { Proporción } \\
\text { ponderada }\end{array}$ & $\mathbf{n}$ & $\begin{array}{l}\text { Proporción } \\
\text { ponderada }\end{array}$ & IC95\% & \\
\hline Total & 4874 & 100,0 & 960 & 24,9 & $22,9-26,9$ & \\
\hline Sexo & & & & & & 0,306 \\
\hline Hombre & 2203 & 47,4 & 446 & 26,0 & $23,0-29,3$ & \\
\hline Mujer & 2671 & 52,6 & 514 & 23,8 & $21,3-26,5$ & \\
\hline Grupo etario (años) & & & & & & 0,640 \\
\hline $60-69$ & 2634 & 52,9 & 558 & 26,1 & $23,5-28,8$ & \\
\hline $70-79$ & 1575 & 30,1 & 308 & 24,1 & $20,6-28,0$ & \\
\hline $80-89$ & 581 & 14,9 & 84 & 22,7 & $16,9-29,8$ & \\
\hline 90 o más & 84 & 2,1 & 10 & 20,3 & $9,0-39,6$ & \\
\hline Estado civil & & & & & & 0,009 \\
\hline Casado(a) o conviviente & 2631 & 53,2 & 552 & 26,5 & $23,9-29,3$ & \\
\hline Soltero & 757 & 16,9 & 157 & 28,6 & $23,4-34,4$ & \\
\hline Viudo(a) o separado(a) & 1486 & 29,9 & 251 & 19,8 & $16,4-23,7$ & \\
\hline Área de residencia & & & & & & $<0,001$ \\
\hline Rural & 2165 & 23,0 & 319 & 13,9 & $12,1-16,0$ & \\
\hline Urbano & 2709 & 77,0 & 641 & 28,1 & $25,7-30,7$ & \\
\hline Nivel educativo & & & & & & $<0,001$ \\
\hline Sin educación o inicial & 1091 & 15,6 & 134 & 12,4 & $9,3-16,3$ & \\
\hline Primaria & 2236 & 29,1 & 346 & 18,1 & $15,6-21,0$ & \\
\hline Secundaria & 934 & 25,8 & 235 & 26,8 & $22,8-31,2$ & \\
\hline Superior & 613 & 19,4 & 245 & 45,8 & $39,9-51,8$ & \\
\hline Afiliado a un seguro de salud & & & & & & $<0,001$ \\
\hline No & 828 & 18,9 & 128 & 16,3 & $12,6-20,9$ & \\
\hline Sí & 4046 & 81,1 & 832 & 26,8 & $24,6-29,2$ & \\
\hline Dominio geográfico & & & & & & $<0,001$ \\
\hline Lima Metropolitana & 547 & 37,3 & 178 & 34,5 & $30,2-39,1$ & \\
\hline Resto de Costa & 1252 & 25,1 & 280 & 22,8 & $20,0-25,8$ & \\
\hline Sierra & 2180 & 27,9 & 376 & 17,9 & $15,6-20,3$ & \\
\hline Selva & 895 & 9,7 & 126 & 13,3 & $10,7-16,4$ & \\
\hline Limitación física & & & & & & 0,606 \\
\hline No & 4437 & 91,9 & 897 & 25,0 & $23,0-27,2$ & \\
\hline Sí & 437 & 8,1 & 63 & 22,8 & $15,9-31,6$ & \\
\hline \multicolumn{7}{|l|}{ Quintiles de bienestar } \\
\hline Quintil inferior & 2047 & 22,5 & 261 & 10,9 & $9,3-12,7$ & $<0,001$ \\
\hline Segundo quintil & 903 & 16,1 & 166 & 16,7 & $13,4-20,6$ & \\
\hline Quintil intermedio & 691 & 16,4 & 128 & 19,3 & $15,3-23,9$ & \\
\hline Cuarto quintil & 644 & 21,1 & 180 & 30,9 & $25,8-36,6$ & \\
\hline Quintil superior & 589 & 23,9 & 224 & 42,1 & $36,9-47,5$ & \\
\hline Idioma / lengua que aprendió en la niñez & & & & & & 0,005 \\
\hline Otros $^{*}$ & 2855 & 71,5 & 611 & 26,6 & $24,2-29,2$ & \\
\hline Quechua y lenguas aborígenes & 2019 & 28,5 & 349 & 20,4 & $17,3-23,9$ & \\
\hline
\end{tabular}

Todos los análisis incluyeron el factor de ponderación y especificaciones muestrales de la ENDES 2018 *Otros: castellano, portugués u otra lengua extranjera IC95\%: intervalo de confianza al 95\% 
Tabla 2. Modelo en dos etapas para el uso de servicios odontológicos por parte de adultos mayores peruanos como variable dependiente, Encuesta Demográfica y de Salud Familiar (ENDES) 2018

\begin{tabular}{|c|c|c|c|c|c|c|}
\hline \multirow{3}{*}{ Variable } & \multicolumn{6}{|c|}{ Factores asociados al uso de servicios odontológicos } \\
\hline & \multicolumn{3}{|c|}{ Modelo crudo } & \multicolumn{3}{|c|}{ Modelo ajustado * } \\
\hline & $\mathbf{R P}$ & IC95\% & Valor de $p$ & $\mathbf{R P a}$ & IC95\% & Valor de $p$ \\
\hline \multicolumn{7}{|l|}{ Sexo } \\
\hline Hombre & Ref. & - & - & - & - & - \\
\hline Mujer & 0,92 & $0,77-1,08$ & 0,305 & - & - & - \\
\hline \multicolumn{7}{|l|}{ Grupo etario (años) } \\
\hline $60-69$ & Ref. & - & - & - & - & - \\
\hline $70-79$ & 0,92 & $0,77-1,11$ & 0,401 & - & - & - \\
\hline $80-89$ & 0,87 & $0,64-1,18$ & 0,368 & - & - & - \\
\hline 90 o más & 0,78 & $0,36-1,67$ & 0,519 & - & - & - \\
\hline \multicolumn{7}{|l|}{ Estado civil } \\
\hline Casado(a) o conviviente & Ref. & - & - & Ref. & - & - \\
\hline Soltero & 1,08 & $0,86-1,35$ & 0,503 & 1,09 & $0,89-1,33$ & 0,397 \\
\hline Viudo(a) o separado(a) & 0,75 & $0,61-0,93$ & 0,007 & 0,85 & $0,70-1,03$ & 0,102 \\
\hline \multicolumn{7}{|l|}{ Área de residencia } \\
\hline Rural & Ref. & - & & Ref. & - & - \\
\hline Urbano & 2,02 & $1,71-2,38$ & $<0,001$ & 0,62 & $0,48-0,80$ & $<0,001$ \\
\hline \multicolumn{7}{|l|}{ Nivel educativo } \\
\hline Sin educación o inicial & Ref. & - & - & Ref. & - & - \\
\hline Primaria & 1,46 & $1,07-1,99$ & 0,016 & 1,17 & $0,85-1,61$ & 0,337 \\
\hline Secundaria & 2,16 & $1,56-2,98$ & $<0,001$ & 1,36 & $0,94-1,99$ & 0,107 \\
\hline Superior & 3,69 & $2,71-5,01$ & $<0,001$ & 1,94 & $1,33-2,83$ & 0,001 \\
\hline \multicolumn{7}{|l|}{ Afiliado a un seguro de salud } \\
\hline No & Ref. & - & - & Ref. & - & - \\
\hline Sí & 1,64 & $1,25-2,15$ & $<0,001$ & 1,58 & $1,23-2,03$ & $<0,001$ \\
\hline \multicolumn{7}{|l|}{ Dominio geográfico } \\
\hline Lima Metropolitana & Ref. & - & - & Ref. & - & - \\
\hline Resto de Costa & 0,66 & $0,55-0,79$ & $<0,001$ & 0,85 & $0,71-1,02$ & 0,077 \\
\hline Sierra & 0,52 & $0,43-0,62$ & $<0,001$ & 0,88 & $0,71-1,09$ & 0,248 \\
\hline Selva & 0,39 & $0,30-0,50$ & $<0,001$ & 0,73 & $0,56-0,95$ & 0,019 \\
\hline \multicolumn{7}{|l|}{ Limitación física } \\
\hline No & Ref. & - & - & - & - & - \\
\hline Sí & 0,92 & $0,64-1,30$ & 0,611 & - & - & - \\
\hline \multicolumn{7}{|l|}{ Quintiles de gasto per cápita } \\
\hline Quintil inferior & Ref. & - & - & Ref. & - & - \\
\hline Segundo quintil & 1,54 & $1,18-2,01$ & 0,002 & 1,97 & $1,47-2,64$ & $<0,001$ \\
\hline Quintil intermedio & 1,77 & $1,35-2,33$ & $<0,001$ & 2,30 & $1,61-3,29$ & $<0,001$ \\
\hline Cuarto quintil & 2,85 & $2,25-3,60$ & $<0,001$ & 3,32 & $2,31-4,77$ & $<0,001$ \\
\hline Quintil superior & 3,87 & $3,17-4,73$ & $<0,001$ & 3,84 & $2,68-5,51$ & $<0,001$ \\
\hline \multicolumn{7}{|c|}{ Idioma / lengua que aprendió en la niñez } \\
\hline Otros & Ref. & - & - & Ref. & - & - \\
\hline Quechua o lenguas aborígenes & 0,77 & $0,63-0,93$ & 0,006 & 1,20 & $0,98-1,47$ & 0,070 \\
\hline
\end{tabular}

*Modelo ajustado por todas las variables que resultaron con un valor de $p<0,2$ en el análisis bivariado RP: Razón de prevalencia, RPa: Razón de prevalencia ajustada, IC95\%: intervalo de confianza al 95\% 
peruana, quienes han mostrado prevalencias de uso de servicios de salud dental por encima del promedio en estudios previos ${ }^{(17)}$, incluso en otros grupos de la población, como en niños menores de 12 años ${ }^{(21)}$. Además, un estudio reciente indica que el uso de servicios en las zonas rurales se ha incrementado luego de la implementación del AUS ${ }^{(22)}$. La razón puede estar en el funcionamiento de algunos programas de transferencia condicionada tales como «JUNTOS» que ofrecen incentivos económicos a población en condición de pobreza o pobreza extrema a cambio de utilizar los servicios de salud ${ }^{23)}$. El fortalecimiento y expansión de programas de este tipo podrían incrementar la utilización de servicios odontológicos en otras regiones de Perú.

El tener un mayor nivel educativo aumentó las probabilidades de utilizar los servicios odontológicos en los AM de nuestro estudio, lo que es consistente con un estudio en AM de Chile, que reportó que aquellos que tuvieron un nivel educativo de secundaria completa o superior tuvieron mayores probabilidades de uso delos servicios odontológicos dentro de un año previo a la aplicación de la encuesta, en comparación con los de menor nivel educativo ${ }^{(24)}$. Al respecto, un mayor nivel educativo está relacionado al nivel de ingresos de una persona, y una mejor economía facilita los recursos para acceder a los servicios de salud, en términos de costo de atención y tratamiento (en el caso de servicios privados), adquisición de medicamentos y transporte hacia el centro de salud, pudiendo favorecer la presencia de desigualdades en el acceso a salud ${ }^{(25)}$. Asimismo, un menor nivel educativo puede denotar una menor conciencia sobre el cuidado de la salud, por lo que resulta fundamental fortalecer mensajes sobre promoción de la salud oral dirigidos a AM, con el fin de crear conciencia e incrementar las probabilidades de uso de servicios odontológicos en esta población ${ }^{(25)}$.

El proveer aseguramiento en salud a la población de AM puede mejorar las oportunidades de uso de los servicios odontológicos ${ }^{(26)}$. Al respecto, es importante señalar, que dos de cada diez participantes en nuestro estudio no se encontraban afiliados a un seguro de salud; asimismo, sólo $16 \%$ de la población de AM sin seguro de salud tuvo atención odontológica durante los seis meses previos a la encuesta. Estos resultados exponen que la falta de aseguramiento en salud reduce las posibilidades de utilizar los servicios de salud públicos en una buena fracción de los AM peruanos, condicionándolos a realizar un mayor gasto de bolsillo para ser atendidos en el sector público o privado. Este problema puede repercutir negativamente sobre la economía del adulto mayor y/o su hogar, teniendo en cuenta que casi $30 \%$ de hogares peruanos tiene como jefe del hogar a un adulto mayor ${ }^{(27)}$, además, cabe señalar que los servicios odontológicos ocupan el cuarto lugar entre los servicios de salud que ocasionan mayor gasto de bolsillo en nuestro país ${ }^{(28)}$.

Los AM pertenecientes a la región selva tuvieron menores probabilidades de uso de servicios de salud dental que aquellos que viven en la costa. Este resultado es consistente con el reporte de la prevalencia en el uso de los servicios de salud oral en AM de la selva peruana el 2014 , que alcanzó un $69,6 \%$, mientras que los de la costa alcanzaron $84,6 \%{ }^{(17)}$. El bajo uso de servicios de salud oral en la selva peruana ocurre incluso en la población de niños menores de 12 años ${ }^{(16)}$.

Una de las razones que explicarían esta disparidad es que, incluso luego del incremento en el uso de servicios de salud oral en la región de la selva posimplementación del AUS en el 2009, la curva de concentración de uso de servicios sigue favoreciendo a la población con mayor poder adquisitivo ${ }^{(22)}$, siendo un problema si se tiene en cuenta que la selva es una de las regiones naturales con más alta proporción de pobladores que viven en condición de pobreza monetaria ${ }^{(29)}$.

Otros factores que afectan las oportunidades de uso de servicios odontológicos pueden ser, la ausencia de servicios de salud y/o profesionales odontólogos en algunas zonas de la selva peruana y la lejanía de los centros de salud. En AM con poca o ninguna autonomía para movilizarse, la lejanía se vuelve un factor determinante para utilizar los servicios de salud dada su alta dependencia de terceros para trasladarse al lugar ${ }^{(30,31)}$.

Las probabilidades de uso de los servicios odontológicos de los AM con mayor nivel de bienestar fueron mayores que las del menor quintil, observándose además un marcado gradiente escalonado en cada categoría inferior de ingresos (40,5\% para el quintil superior y $10 \%$ para el quintil inferior). Las razones tras este hallazgo pueden ser explicadas pues la población con menor poder adquisitivo tiene limitado acceso a tratamientos de rehabilitación oral que no son cubiertos por el PEAS, pues suelen ser más complejos y costosos. Asimismo, el Plan Nacional «Vuelve a Sonreír» encargado de rehabilitar a la población de AM peruanos en situación de pobreza y pobreza extrema ha presentado un periodo considerable de inactividad, lo que ha podido generar una disminución de las probabilidades de acceder a este tipo de tratamientos en los AM con menores ingresos.

Por otro lado, sólo cuatro de cada diez AM de 60 años se encuentran afiliados a algún tipo de sistema de pensiones ${ }^{(27)}$, y estas, muy raras veces superan el sueldo mínimo vital nacional, reduciendo las opciones de uso de los servicios odontológicos para este grupo de la población. Además, se debe tener en cuenta que el gasto de bolsillo en salud aumenta con la edad por la presencia de comorbilidades, discapacidades y otros problemas crónicos de salud en los AM empeorando las oportunidades de acceso, sobre todo a la salud dental, donde gran parte de los tratamientos sólo son realizados en el ámbito privado ${ }^{(32,33)}$. Estos hallazgos son consecuentes con un estudio reciente donde se reporta que la brecha de desigualdad en el uso de los servicios 
de salud oral en AM peruanos se está incrementando en los últimos años en favor del grupo de la población con mayores ingresos ${ }^{(22)}$.

Una de las limitaciones del estudio es el uso de bases de datos de la ENDES para llevar a cabo el análisis, en cuyo proceso de recolección de información, el encuestado pudo incurrir en sesgos de memoria dada la naturaleza de autorreporte de la variable principal de estudio. Además, debido a que la base de datos no contiene información de la necesidad de tratamiento, no podemos conocer en qué medida esta variable influye en el uso de los servicios odontológicos en la población de AM peruanos. Asimismo, el diseño transversal del estudio primario no nos permite evaluar relaciones de causalidad. Pese a las limitaciones, el presente estudio evalúa los factores asociados al uso de los servicios de salud dental empleando los datos de la ENDES, que ofrecen un nivel de representatividad nacional, y emplea cuestionarios estandarizados para el proceso de recolección de la información, reduciendo la posibilidad de sesgo de información.

En conclusión, el uso de los servicios odontológicos en la población de AM es bajo y no ha presentado mejoras en los últimos años. Asimismo, sus factores asociados fueron el área de residencia, el nivel educativo, la afiliación a un seguro de salud, el dominio geográfico y el quintil de bienestar. En ese sentido, la salud oral del adulto mayor en el Perú requiere de una reforma que tome en cuenta las características reportadas como asociadas en nuestro estudio y similares.
Aunque se requiere de estudios futuros para confirmar nuestras hipótesis, podemos inferir que las desigualdades identificadas responden a diversos aspectos, entre los que destacan, la limitada cartera de tratamientos de salud oral en los servicios públicos de salud, la desactivación prolongada de planes como «Vuelve a Sonreír» orientados a tratar las necesidades no cubiertas en el sistema de salud público en población pobre y pobre extrema, el número considerable de $\mathrm{AM}$ peruanos aún sin seguro de salud y la falta de campañas de promoción de la salud oral dirigidas a AM; todas ellas, convergen en la falta de recursos de la población para acceder a tratamientos oportunos y de calidad más allá de los establecimientos privados, generando un gasto de bolsillo considerable. Por ello, la instauración de mecanismos que contrarresten el problema del uso de los servicios odontológicos debe ser una de las prioridades del Gobierno a través del Ministerio de Salud del Perú, de lo contrario, la salud oral seguirá siendo un privilegio de las personas con mayor capacidad de gasto.

Contribuciones de autoría: CRR y RVF realizaron el análisis y verificaron los métodos analíticos. DA redactó la primera versión del manuscrito con aportaciones de todos los autores. Todos los autores discutieron los resultados, contribuyeron al manuscrito final y asumen responsabilidad frente al contenido del manuscrito.

Agradecimientos: a Akram Hernández-Vásquez quién ideó el estudio y participó en el análisis estadístico.

Fuentes de financiamiento: autofinanciado.

Conflictos de interés: los autores declaran no tener conflictos de interés.

\section{REFERENCIAS BIBLIOGRÁFICAS}

1. Fondo de Población de las Naciones Unidas. Adultos Mayores en el Perú [Internet]. Lima: UNFPA; 2016 [citado 10 de agosto de 2019]. Disponible en: https://peru.unfpa.org/sites/default/files/pub-pdf/UNFPA-Ficha-Adultos-Mayores-Peru.pdf

2. Fukai $\mathrm{K}$, Ogawa $\mathrm{H}, \mathrm{Hescot} \mathrm{P}$. Oral health for healthy longevity in an ageing society: maintaining momentum and moving forward. Int Dent J. 2017;67 Suppl 2:3-6. doi:10.1111/idj.12347.

3. Azevedo MS, Correa MB, Azevedo JS, Demarco FF. Dental prosthesis use and/or need impacting the oral healthrelated quality of life in Brazilian adults and elders: Results from a National Survey. J Dent. 2015;43(12):1436-41. doi:10.1016/j.jdent.2015.10.016.

4. Masood M, Newton T, Bakri NN, Khalid T, Masood Y. The relationship between oral health and oral health related quality of life among elderly people in United
Kingdom. J Dent. 2017;56:78-83. doi:10.1016/j.jdent.2016.11.002.

5. Tonetti MS, Jepsen S, Jin L, Otomo-Corgel J. Impact of the global burden of periodontal diseases on health, nutrition and wellbeing of mankind: A call for global action. J Clin Periodontol. 2017;44(5):456 62. doi:10.1111/jcpe. 12732 .

6. Oral health inequalities: A major public health challenge for Asia-Pacific. Nature India; 2017 [citado 10 de agosto de 2019]. Disponible en: https://www.natureasia. com/en/nindia/article/10.1038/ nindia.2017.21

7. Fisher J, Selikowitz H-S, Mathur M Varenne B. Strengthening oral health for universal health coverage. Lancet. 2018;392(10151):899-901. doi:10.1016/ S0140-6736(18)31707-0.

8. Ministerio de Salud. Plan Esencial de Aseguramiento En Salud (PEAS). Plan de Beneficios Del PEAS [Internet]. Lima:
MINSA; 2009 [citado 11 de agosto de 2019]. Disponible en: http://www. minsa.gob.pe/portada/aseguramiento/ archivo/PEAS.pdf

9. Pengpid S, Peltzer K. The prevalence of edentulism and their related factors in Indonesia, 2014/15. BMC Oral Health. 2018;18(1):118.doi:10.1186/s12903-0180582-7.

10. Olofsson H, Ulander EL, Gustafson Y, Hörnsten C. Association between socioeconomic and health factors and edentulism in people aged 65 and older - a population-based survey. Scand J Public Health. 2018;46(7):690-8. doi:10.1177/1403494817717406.

11. Ministerio de Salud. Plan de Intervención Para La Rehabilitación Oral Con Prótesis Removible en el Adulto Mayor 2012-2016 «Vuelve a Sonreir». Lima: MINSA; 2011. [citado 11 de agosto de 2019]. Disponible en: http://bvs.minsa. gob.pe/local/MINSA/2409.pdf 
12. Ministerio de Salud. Plan Nacional de Atención Integral para la Rehabilitación Oral en Personas Adultas Mayores «Perú Sonríe» 2019-2021 [Internet]. Lima: MINSA; 2019. [citado 10 de octubre de 2019] Disponible en: https:// cdn.www.gob.pe/uploads/document/ file/340422/Resoluci\%C3\%B3n Ministerial_N_619-2019-MINSA.pdf

13. Lee W, Kim S-J, Albert JM, Nelson S. Community factors predicting dental care utilization among older adults. J Am Dent Assoc. 2014;145(2):150-8. doi:10.14219/jada.2013.22.

14. Almutlaqah MA, Baseer MA, Ingle NA, Assery MK, Al Khadhari MA. Factors Affecting Access to Oral Health Care among Adults in Abha City, Saudi Arabia. J Int Soc Prev Community Dent. 2018;8(5):431-8. doi:10.4103/jispcd. JISPCD_205_18.

15. Silva AER, Langlois $\mathrm{C}$ de O, Feldens CA. Use of dental services and associated factors among elderly in southern Brazil. Rev Bras Epidemiol. 2013;16(4):1005-16. doi:10.1590/S1415790X2013000400020.

16. Azañedo D, Hernández-Vásquez A, Casas-Bendezú M, Gutiérrez C, AgudeloSuárez AA, Cortés $S$. Factors determining access to oral health services among children aged less than 12 years in Peru. F1000Res. 2017;6:1680. doi:10.12688/ f1000research.12474.1.

17. Azañedo D, Díaz-Seijas D, HernándezVásquez A. Salud oral del adulto mayor: acceso, desafíos y oportunidades para la salud pública peruana. Rev Peru Med Exp Salud Publica. 2016;33(2):373-375.

18. Ley de la Persona Adulta Mayor, Ley No.30490. Diario El Peruano. 20 julio 2016. [citado 12 de agosto de 2019]. Disponible en: https:// busquedas.elperuano.pe/normaslegales/ ley-de-la-persona-adulta-mayorley-n-30490-1407242-1/

19. Barros AJ, Hirakata VN. Alternatives for logistic regression in cross-sectional studies: an empirical comparison of models that directly estimate the prevalence ratio. BMC Med Res Methodol. 2003;3:21.

20. Bursac Z, Gauss CH, Williams DK, Hosmer DW. Purposeful selection of variables in logistic regression. Source Code Biol Med. 2008;3:17.

21. Hernández-Vásquez A, Azañedo D, Díaz-Seijas D, et al. Acceso a servicios de salud dental en menores de doce años en Perú, 2014. Salud Colect. 2016;12(3):429-441. doi:10.18294/ sc.2016.912.

22. Hernández-Vásquez A, Bendezu-Quispe G, Azañedo D, Santero M. Use of oral health care services in Peru: trends of socio-economic inequalities before and after the implementation of Universal Health Assurance. BMC Oral Health. 2019;19(1):39. doi:10.1186/s12903019-0731-7.

23. Aramburú CE. Informe compilatorio: el Programa Juntos, resultados y retos 2010 [citado 12 de agosto de 2019]. Disponible en: https://books.google.com/ books/about/Informe_compilatorio.ht$\mathrm{ml}$ ?hl=\&id=U-YktwAACAAJ

24. Mariño R, Giacaman RA. Patterns of use of oral health care services and barriers to dental care among ambulatory older Chilean. BMC Oral Health. 2017;17(1):38. doi:10.1186/s12903-016-0329-2.

25. Hahn RA, Truman BI. Education Improves Public Health and Promotes Health Equity. Int J Health Serv. 2015;45(4):657678. doi:10.1177/0020731415585986.

26. Kreider B, Manski RJ, Moeller J, Pepper $\mathrm{J}$. The effect of dental insurance on the use of dental care for older adults: a partial identification analysis. Health Econ. 2015;24(7):840-58. doi:10.1002/ hec.3064.

27. Instituto Nacional de Estadística e Informática. Situación de La Población Adulta Mayor [Internet]. Lima: INEI; 2018. [citado 15 de agosto de 2019]. Disponible en: https://www.inei.gob.pe/ media/MenuRecursivo/boletines/01- informe-tecnico-n02_adulto_ene-feb_ mar2018.pdf

28. Proaño Falconi D, Bernabé E. Determinants of catastrophic healthcare expenditure in Peru. Int J Health Econ Manag. 2018. doi:10.1007/s10754-018-9245-0.

29. Instituto Nacional de Estadística e Informática. Informe Técnico: Evolución de la Pobreza Monetaria 2007-2017 [Internet]. Lima: INEI; 2017. [citado 15 de agosto de 2019]. Disponible en: https:// www.inei.gob.pe/media/cifras_de pobreza/informe_tecnico_pobreza_ monetaria_2007-2017.pdf

30. Montini T, Tseng T-Y, Patel H, Shelley D. Barriers to dental services for older adults. Am J Health Behav. 2014;38(5):781-8. doi:10.5993/AJHB.38.5.15.

31. Bharti R, Chandra A, Tikku AP, Arya D, Gupta R. Oral care needs, barriers and challenges among elderly in India. J Indian Prosthodont Soc. 2015;15(1):17-22. doi:10.4103/09724052.155044 .

32. Hernández-Vásquez A, Rojas-Roque C, Santero M, Prado-Galbarro FJ, Rosselli D. Gasto de bolsillo en salud en adultos mayores peruanos: análisis de la encuesta nacional de hogares sobre condiciones de vida y pobreza 2017. Rev Peru Med Exp Salud Publica. 2018;35(3):390-399. doi:10.17843/ rpmesp.2018.353.3815.

33. Hazra NC, Rudisill C, Gulliford MC. Determinants of health care costs in the senior elderly: age, comorbidity, impairment, or proximity to death? Eur J Health Econ. 2018;19(6):831-42. doi:10.1007/s10198-017-0926-2.

\footnotetext{
Correspondencia: Diego Azañedo

Dirección: Urb. El Trapecio B-2 Primera etapa, Chimbote, Perú

Teléfono: (+51) 947121972

Correo electrónico:dazanedov@uladech.edu.pe
} 